\title{
Favorable perioperative outcomes after resection of borderline resectable pancreatic cancer treated with neoadjuvant stereotactic radiation and chemotherapy compared with upfront pancreatectomy for resectable cancer
}

\author{
Eric A. Mellon ${ }^{1}$, Tobin J. Strom ${ }^{1}$, Sarah E. Hoffe ${ }^{1}$, Jessica M. Frakes ${ }^{1}$, Gregory M. Springett ${ }^{2}$, Pamela J. \\ Hodul $^{2}$, Mokenge P. Malafa ${ }^{2}$, Michael D. Chuong ${ }^{3}$, Ravi Shridhar ${ }^{4}$ \\ ${ }^{1}$ Department of Radiation Oncology, ${ }^{2}$ Gastrointestinal Tumor Program, H. Lee Moffitt Cancer Center and Research Institute, Tampa, FL, USA; \\ ${ }^{3}$ Department of Radiation Oncology, University of Maryland, Baltimore, MD, USA; ${ }^{4}$ Florida Hospital Cancer Institute, Orlando, FL, USA \\ Contributions: (I) Conception and Design: EA Mellon, SE Hoffe, R Shridhar; (II) Administrative support: None; (III) Provision of study materials or \\ patients: SE Hoffe, GM Springett, PJ Hodul, MP Malafa, R Shridhar; (IV) Collection and assembly of data: EA Mellon, TJ Strom, JM Frakes, MD \\ Chuong; (V) Data analysis and interpretation: EA Mellon, SE Hoffe, GM Springett, R Shridhar; (VI) Manuscript writing: All authors; (VII) Final \\ approval of manuscript: All authors.
}

Correspondence to: Ravi Shridhar, MD, PhD. 2501 N. Orange Ave, Suite 181, Orlando, FL 32804, USA. Email: ravi0421@yahoo.com.

\begin{abstract}
Background: Neoadjuvant multi-agent chemotherapy and stereotactic body radiation therapy (SBRT) are utilized to increase margin negative (R0) resection rates in borderline resectable pancreatic cancer (BRPC) or locally advanced pancreatic cancer (LAPC) patients. Concerns persist that these neoadjuvant therapies may worsen perioperative morbidities and mortality.

Methods: Upfront resection patients $(n=241)$ underwent resection without neoadjuvant treatment for resectable disease. They were compared to BRPC or LAPC patients $(n=61)$ who underwent resection after chemotherapy and 5 fraction SBRT. Group comparisons were performed by Mann-Whitney U or Fisher's exact test. Overall Survival (OS) was estimated by Kaplan-Meier and compared by log-rank methods.

Results: In the neoadjuvant therapy group, there was significantly higher $\mathrm{T}$ classification, $\mathrm{N}$ classification, and vascular resection/repair rate. Surgical positive margin rate was lower after neoadjuvant therapy $(3.3 \%$ vs. $16.2 \%, \mathrm{P}=0.006)$. Post-operative morbidities (39.3\% vs. $31.1 \%, \mathrm{P}=0.226)$ and 90 -day mortality (2\% vs. $4 \%$, $\mathrm{P}=0.693)$ were similar between the groups. Median OS was 33.5 months in the neoadjuvant therapy group compared to 23.1 months in upfront resection patients who received adjuvant treatment $(\mathrm{P}=0.057)$.

Conclusions: Patients with BRPC or LAPC and sufficient response to neoadjuvant multi-agent chemotherapy and SBRT have similar or improved peri-operative and long-term survival outcomes compared to upfront resection patients.
\end{abstract}

Keywords: Radiotherapy; neoadjuvant therapy; pancreatic neoplasms

Submitted Jan 29, 2016. Accepted for publication Feb 24, 2016.

doi: 10.21037/jgo.2016.03.15

View this article at: http://dx.doi.org/10.21037/jgo.2016.03.15

\section{Introduction}

Pancreatic cancer is currently the fourth most common cause of cancer death in the USA, and is projected to become the second most common by 2030 (1). Long-term survival is less than $2 \%$ in unresectable adenocarcinoma patients (2). However, approximately $15 \%$ of patients present with upfront resectable cancer, and 5-year survival as high as $31.4 \%$ is observed after standard of care pancreatectomy and adjuvant chemotherapy. Therefore, some efforts to improve survival in pancreatic cancer involve expanding the pool of patients who undergo 


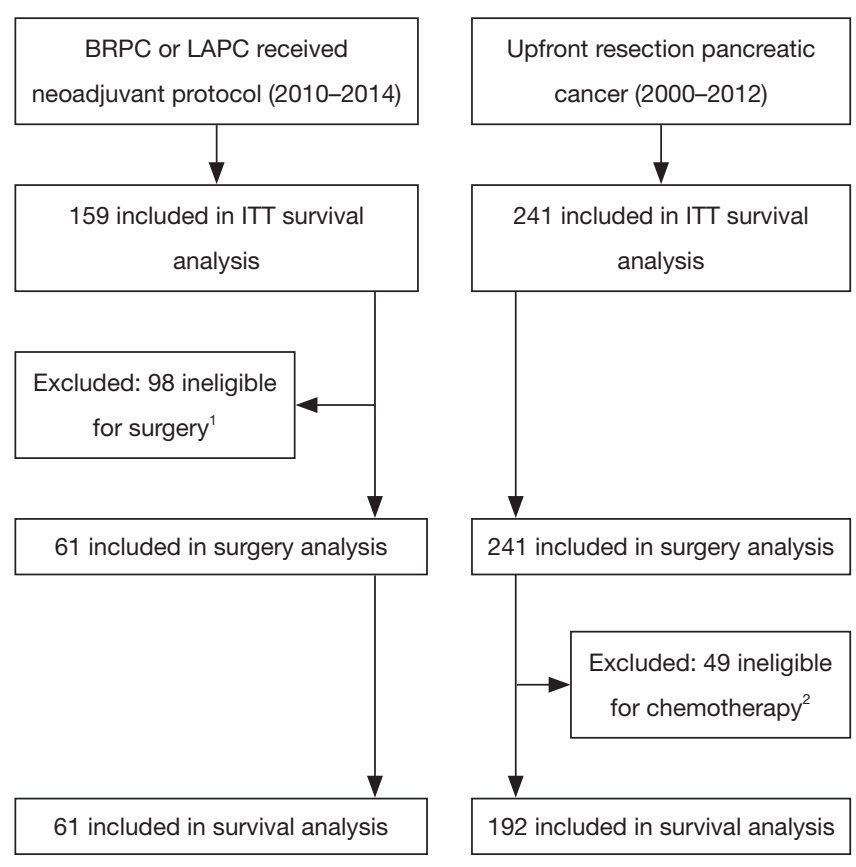

Figure 1 Flow diagram for this study. ${ }^{1}$, of 98 patients ineligible for surgery, there were 44 LAPC patients with insufficient imaging response to neoadjuvant therapy to consider surgery. For the remaining staged as BRPC, 23 progressed on imaging to unresectable, 15 had metastases found at time of surgery, 8 were locally unresectable at time of surgery, and 8 had performance status decline during neoadjuvant therapy making them ineligible for surgery. ${ }^{2}$, due to post-operative performance status or mortality 39 patients were not offered adjuvant therapy, 5 patients refused, and 5 patients were lost to follow-up.

complete tumor resection. This must be balanced with caution, as margin positive resection is associated with a poor prognosis (3). Thus, a subset of patients has been termed borderline resectable pancreatic cancer (BRPC), implying the possibility of margin negative resection after neoadjuvant therapy (4).

Numerous single institution studies have investigated neoadjuvant treatments for BRPC with no current standard of care (5). A 2011 meta-analysis of 14 prospective trials of neoadjuvant chemoradiotherapy with conventional radiation and assorted chemotherapies found that about one-third of tumors initially marginal for resection were ultimately resected (6). Given the lack of randomized trials, two groups have reported survival benefit for mixed populations of resectable and BRPC patients receiving neoadjuvant therapy compared to upfront resection $(7,8)$. Others have reported equivalent survival (9-12).
An alternate neoadjuvant approach utilizes chemotherapy in sequential fashion with stereotactic body radiation therapy (SBRT) - a short course of high dose, precisely focused radiotherapy that might overcome the radioresistance of pancreatic adenocarcinoma. Single institutions have reported margin negative resection rates above $90 \%$ and minimal serious radiation associated toxicity $(13,14)$.

There is continued apprehension about applying any type of radiotherapy before pancreatectomy. Analysis of the American College of Surgeons National Surgical Quality Improvement Program database revealed increased 30-day mortality after pancreaticoduodenectomy among patients who received preoperative radiation therapy (15). One case series demonstrated a $37.5 \%$ rate of portal vein (PV) stenosis after neoadjuvant chemoradiotherapy and pancreatectomy (16). Further, early studies of SBRT and single agent chemotherapy in locally advanced pancreatic cancer (LAPC) without pancreatectomy reported intolerably high acute and late toxicity rates not supported by more recent studies $(17,18)$. To explore whether this intensified regimen worsened perioperative or long-term outcomes, we compared resectable patients who underwent upfront resection to patients resected after sufficient response following neoadjuvant treatment with SBRT and chemotherapy.

\section{Methods}

\section{Initial staging and patients}

Figure 1 summarizes the analysis groups. After institutional review board (IRB) approval, all patients at our institution who underwent curative intent surgery between 2000 and 2012 for pancreatic adenosquamous or adenocarcinoma without neoadjuvant chemotherapy or radiation therapy were identified ("upfront resection" group). Tumors were deemed resectable by the surgeon and not assigned a clinical stage (TX) or were American Joint Committee on Cancer (AJCC) $7^{\text {th }}$ edition stage I-IIB.

Our IRB approved registry contained 159 patients from 2010 to 2014 who received neoadjuvant or definitive chemotherapy and SBRT for the treatment of BRPC or LAPC, AJCC clinical stage IIA-III. 110 BRPC patients received neoadjuvant therapy and $56(51 \%)$ underwent resection. An additional 5 (10\%) of 49 LAPC patients underwent resection after sufficient response to the neoadjuvant treatment regimen. Demographics and outcomes for all BRPC and LAPC patients are reported 
separately (14). As our goal was to analyze the outcomes of surgically resected pancreatic cancer with or without neoadjuvant therapy, patients who did not undergo a curative intent resection were excluded from all analyses except the "intention-to-treat" survival analysis. Resected BRPC and LAPC patients comprise the "neoadjuvant therapy" group.

Staging methods at our institution have been previously described (19). BRPC and LAPC patients were initially judged medically fit to tolerate resection without probable distant metastases. Patients routinely undergo physical examination, standard blood chemistries including CA19-9, multidetector thin-slice pancreatic protocol computed tomography (CT) scan, positron emission tomography (PET) scan, endoscopic ultrasound (EUS), specialist pathology review, and presentation at multi-disciplinary tumor board. The highest stage identified by any imaging modality determined the clinical stage. In the upfront resection group, imaging methods were not standardized, but contrast CT and PET/CT were routinely performed.

\section{Neoadjuvant treatment and restaging}

Chemotherapy and SBRT protocols have been described in detail (20). In summary, patients typically received chemotherapy with three 21-day cycles of gemcitabine, docetaxel, and capecitabine (GTX) for BRPC or six 14-day cycles of oxaliplatin, irinotecan, fluorouracil, and leucovorin (FOLFIRINOX) for LAPC. Other chemotherapies were occasionally given at the discretion of the medical oncologist.

Before radiation planning, 2 to 4 fiducial markers were placed into the tumor under EUS guidance (21). A tumor motion study was then performed to gauge the amplitude of tumor motion by fluoroscopic tracking of the markers during respiration with an applied abdominal compression device. After immobilization by BodyFix cradle (Elekta, Stockholm, Sweden), isocenter was determined on non-contrast CT simulation scan followed by repeat 4-dimensional CT with intravenous and oral contrast. For tumor motion $<1 \mathrm{~cm}$, internal target volume (ITV) and planning organ-at-risk volumes (PRV) encompassing the entire respiratory cycle were used. If tumor motion was $\geq 1 \mathrm{~cm}$, radiation treatment required respiratory gating by the Real-Time Position Management system (Varian Medical Systems Inc., Palo Alto, CA, USA), and treatment was planned and delivered during the $40-60 \%$ respiratory phases with voluntary breath-hold technique.

Dose painting intensity modulated radiation therapy was used to plan 5 fractions daily delivery totaling $30 \mathrm{~Gy}$ to the planning tumor volume (PTV) and concurrently up to 50 Gy to the high dose PTV (hdPTV) as limited by PRV constraints. The PTV consists of 3-5 mm expansion of the ITV generated from gross tumor volume within the pancreas plus motion. The hdPTV is delineated based on CT and EUS to include areas of tumor involvement of vascular structures that limit resectability: celiac axis, SMA, common hepatic artery, gastroduodenal artery, superior mesenteric vein (SMV), PV, or inferior vena cava. Highest priority is given to PRV constraints as follows: each of duodenum, small bowel, and stomach mean $<20 \mathrm{~Gy}$, volume receiving 30 Gy $(V 30)<2$ cc, V35 Gy $<0.5$ cc; each kidney mean $<10$ Gy, spinal cord maximum 20 Gy. SBRT delivered by Varian Truebeam or Trilogy linear accelerator began at least 7 days after chemotherapy.

After completion of neoadjuvant treatment, BRPC and LAPC patients underwent restaging by repeat examination, pancreas protocol CT, and PET/CT. Resectability was again determined at multidisciplinary tumor board. The target time to surgery was 1-2 months after SBRT, and all patients except one underwent surgery within 3 months after SBRT.

\section{Surgery and pathology}

Anatomic resectability definitions were applied from the 2009 expert consensus statement except in the upfront resection group prior to 2009 when resectable was defined by the surgeon (4). Patients with pancreatic head tumors underwent pancreaticoduodenectomy with pylorus sparing based on evolving surgeon preference over the study period. Those with pancreatic body or tail tumors underwent distal pancreatectomy and splenectomy. A small percentage of patients required total pancreatectomy due to concerns of diffuse tumor infiltration or numerous intraductal mucinous papillary neoplasms. A vascular surgeon was available in case of need for repair or resection of the SMV or PV.

College of American Pathology protocols were used for pathologic examination, including tumor response grade to neoadjuvant therapy. Grading was performed by pathologists with expertise in gastrointestinal pathology. Margin negative was defined as no tumor cells at the specimen edge.

\section{Follow-up and analysis}

All charts were reviewed to identify post-operative morbidity, mortality, adjuvant treatment, and toxicities. Side effects were graded according to version 4.0 of the National 
Table 1 Patient characteristics and neoadjuvant treatments

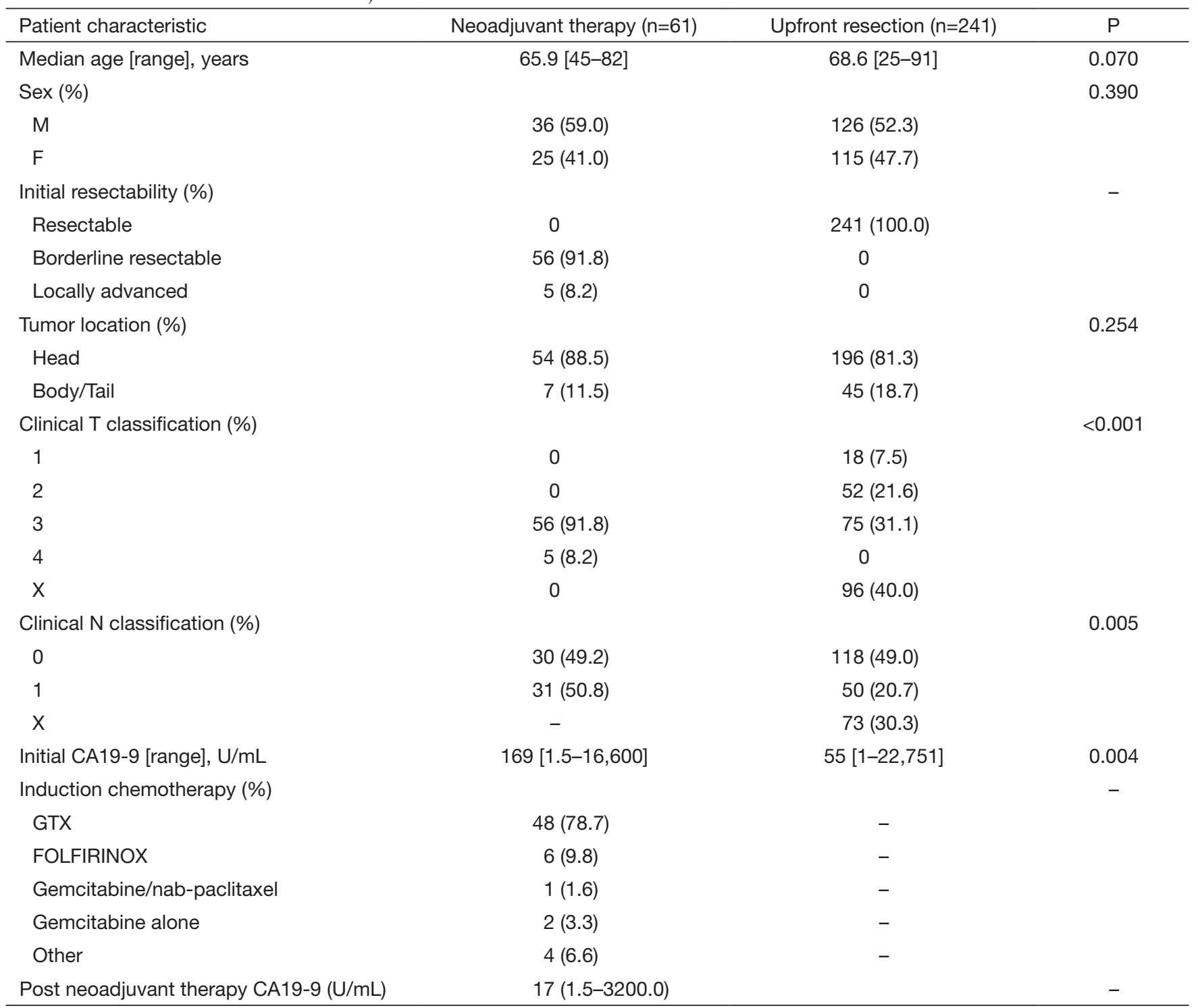

GTX, gemcitabine, docetaxel, capecitabine; FOLFIRINOX, oxaliplatin, irinotecan, fluorouracil, and leucovorin.

Cancer Institute Common Terminology Criteria for Adverse Events. Patients were followed every 3-6 months for the first 5 years and yearly thereafter.

Statistical testing was performed using IBM SPSS (Windows Version 20.0; IBM Corp, Armonk, NY, USA). Comparisons between groups were performed by 2 -sided exact Mann-Whitney test for continuous variables or 2 -sided Fisher's exact test for nominal variables. Survival was estimated by Kaplan-Meier method and compared by log-rank (Mantel-Cox) test, with time starting at the first cancer directed treatment (chemotherapy or surgery).

\section{Results}

Patient characteristics and neoadjuvant treatments for the surgery analysis group (see Figure 1) are summarized in Table 1. The neoadjuvant therapy group had higher $\mathrm{T}$ classification and $\mathrm{N}$ classification compared to upfront resection $(\mathrm{P}<0.001$ and $\mathrm{P}=0.005$, respectively; unknowns excluded).

Within the surgery analysis group, grade $\geq 3$ toxicities occurred in 3 patients $(4.9 \%)$ that were potentially attributable to SBRT. One patient had grade 3 duodenal 
Table 2 Surgery characteristics and complications for all patients

\begin{tabular}{|c|c|c|c|}
\hline Surgical details and outcomes & Neoadjuvant therapy $(n=61)$ & Upfront resection $(n=241)$ & $\mathrm{P}$ \\
\hline Surgery type & & & 0.275 \\
\hline Whipple \pm pylorus sparing & $54(88.5)$ & $194(80.5)$ & \\
\hline Total pancreatectomy & $1(1.6)$ & $4(1.7)$ & \\
\hline SMV or PV resection or repair & $22(36.1)$ & $19(7.9)$ & $<0.001$ \\
\hline No vein resection/repair & $400[100-1,500]$ & $300[50-2,000]$ & 0.247 \\
\hline Vein resection/repair & $500[200-4,000]$ & $400[50-1,500]$ & 0.298 \\
\hline Median surgery time [range], $\mathrm{h}$ & 9 [6-13] & 8 [4-15] & 0.002 \\
\hline No vein resection/repair & 9 [6-13] & 8 [4-15] & 0.014 \\
\hline Vein resection/repair & 10 [8-13] & $12[8-14]$ & 0.058 \\
\hline Vein resection/repair & $11[8-27]$ & $12.5[6-84]$ & 0.152 \\
\hline Post-operative complications & $24(39.3)$ & $75(31.1)$ & 0.226 \\
\hline Pancreatic or biliary leak & $10(16.4)$ & $28(12.0)$ & 0.423 \\
\hline Wound infection & $10(16.4)$ & $26(11.6)$ & 0.267 \\
\hline Wound dehiscence or fistula & $3(4.9)$ & $3(1.2)$ & 0.198 \\
\hline Abscess & $4(6.6)$ & $10(4.1)$ & 0.493 \\
\hline Internal bleeding & 0 & $9(3.7)$ & 0.212 \\
\hline Stricture & $1(1.6)$ & $1(0.4)$ & 0.364 \\
\hline Peritonitis & $1(1.6)$ & $4(1.7)$ & 1.000 \\
\hline
\end{tabular}

Whipple, pancreaticoduodenectomy; SMV, superior mesenteric vein; PV, portal vein; DVT, deep venous thrombosis; PE, pulmonary embolism.

bleed associated with tumor invasion and ulceration 3 weeks after SBRT. The patient underwent endoscopic cauterization and pancreaticoduodenectomy soon afterwards with uneventful post-operative course. One patient suffered biliary ductal stenosis 7 months after pancreaticoduodenectomy and underwent repeat pancreaticojejunostomy. Another patient experienced portal hypertension and malabsorption resulting from portal venous stricture 18 months after pancreaticoduodenectomy. Grade $\geq 3$ toxicities occurred in 26 patients $(42.6 \%)$ that were potentially attributable to neoadjuvant chemotherapy. Most toxicity was hematologic as neutropenia $(n=15)$, anemia $(n=4)$, or thrombocytopenia $(n=2)$. Twelve $(19.7 \%)$ patients experienced at least one grade $3+$ symptomatic toxicity due to chemotherapy, including vomiting $(n=3)$, febrile neutropenia $(n=2)$, palmar-plantar erythrodysesthesia $(n=2)$, oral mucositis $(n=2)$, diarrhea $(n=2)$, toxic epidermal necrolysis $(\mathrm{n}=1)$, and hypertension $(\mathrm{n}=1)$.

Table 2 demonstrates no differences in the documented post-operative morbidities listed (all $\mathrm{P}>0.1$ ). The overall rate of a patient experiencing surgical morbidity was $39.3 \%$ in the neoadjuvant therapy group and $31.1 \%$ in the upfront resection group $(\mathrm{P}=0.226)$. There was less estimated blood loss (EBL) and shorter surgery time in the upfront resection group, while neoadjuvant patients had shorter hospitalization times. However, when stratified for vein resection or repair, hospitalization and EBL were not statistically different. Median surgery time for patients not requiring vascular repair remained $1 \mathrm{~h}$ longer in the 
neoadjuvant therapy group.

The positive margin rate was only $3.3 \%$ after neoadjuvant therapy compared to $16.2 \%$ with upfront resection $(\mathrm{P}=0.006)$. Table 3 further reports that the median pathologic size of the primary was smaller with neoadjuvant therapy (2.5 vs. $3.0 \mathrm{~cm}, \mathrm{P}<0.001)$ and the pathologic $\mathrm{T}$ classification was lower $(\mathrm{P}=0.010)$ with 4 pathologic complete responses $(6.6 \%)$. Despite more lymph nodes resected in the neoadjuvant therapy group (20 vs. 12 , $\mathrm{P}<0.001$ ), there was a trend towards fewer positive lymph nodes in the neoadjuvant therapy group ( 2 vs. $3, \mathrm{P}=0.054)$.

Comparisons of overall survival (OS) are presented in Figure 2. The intention-to-treat survival analysis (Figure $2 A$ ) includes all 159 BRPC and LAPC patients treated with neoadjuvant therapy (61 resected, 98 not resected). As expected, these mostly unresected pancreatic adenocarcinoma patients had worse survival than those who underwent upfront resection (median OS 17.0 vs. 22.1 months, $\mathrm{P}=0.029$ ).

Of the 241 upfront resection patients, 45 (18.7\%) received adjuvant chemotherapy alone and 147 (61.0\%) received chemoradiation guided by Radiation Therapy Oncology Group 97-04 protocol (22). To compare outcomes for patients who received comparable treatment (surgery and at least neoadjuvant or adjuvant chemotherapy), we excluded from further survival analysis the remaining $49(20.3 \%)$ who received no adjuvant treatment or were lost to follow-up, leaving 192 patients. For the survival analysis, the neoadjuvant therapy group includes the 61 who were resected.

Thus in the survival analysis group (Figure $2 B$ ), the estimated median OS for the neoadjuvant therapy group ( $\mathrm{n}=61$, median follow-up 18.1 months) was $33.5 \mathrm{vs}$. 23.1 months in the upfront resection group ( $\mathrm{n}=192$, median follow-up 22.2 months) (Figure $2 B, \mathrm{P}=0.057$ ). Locoregional failures, isolated or concurrent with first distant metastasis, were observed in 5 of $61(8.2 \%)$ neoadjuvant therapy patients and in 22 of 192 (11.5\%) upfront resection patients ( $\log \operatorname{rank} \mathrm{P}=0.786)$.

To attempt to control for baseline differences between groups, univariate and multivariate analyses for OS are presented in the Supplemental materials. On multivariate analysis, no baseline characteristic was significant for survival, but trends remained for neoadjuvant therapy group ( $\mathrm{P}=0.077)$ and $\mathrm{N}$ classification $(\mathrm{P}=0.091)$. To attempt to control for year of therapy, a matched analysis was performed for patients who underwent treatment between 2010 and 2012. Margin positive rates remained significantly different within neoadjuvant therapy $(n=41)$ and upfront resectable $(\mathrm{n}=59)$ cohorts $(2 \%$ vs. $16 \%, \mathrm{P}=0.029)$. OS was similar (33.5 vs. 22.9 months, $\mathrm{P}=0.317)$.

\section{Discussion}

Because complete resection is the only curative option for pancreatic adenocarcinoma, we have applied an aggressive neoadjuvant regimen of SBRT and multi-agent chemotherapy with the goal of resection of cancers initially marginal or ineligible for resection. These techniques could induce unacceptable surgical results or toxicity, and so this work analyzed outcomes for neoadjuvantly treated patients compared to those presenting with resectable pancreas cancer who underwent upfront resection without neoadjuvant chemotherapy or radiation. We found no evidence for worsened surgical or survival outcomes due to neoadjuvant therapy.

A superior negative margin rate for BRPC and LAPC treated neoadjuvantly was observed despite more use of vascular repair and higher initial clinical $\mathrm{T}$ and $\mathrm{N}$ classifications. The margin positive rates in this study are encouraging compared to other published studies of BRPC, though there is significant variability ( $2 \%$ to $89 \%$ ) based on criteria for resectability and neoadjuvant regimen $(23,24)$. Previously, acceptable negative margin rates and toxicities have been observed after neoadjuvant chemotherapy and SBRT in BRPC $(13,20)$.

The benefits of neoadjuvant therapy are partially explained by selection of patients who have good response to chemoradiotherapy prior to surgery. Attempts were made to control for other group differences, such as differences in staging and resectability definitions, by multivariate and matched time period analyses. In addition, we attempted to control for lead time bias by including in the survival analysis only upfront resection patients who received adjuvant therapy. As this study presents a non-randomized comparison of two retrospective cohorts, there may be unrecognized confounding factors that were not anticipated. For example, while pre-operative CA19-9 levels have had mixed prognostic value in pancreatic cancer, pre-operative CA19-9 was difficult to interpret in this series due to confounding from preoperative biliary obstruction that was managed differently prior to surgery in the two cohorts $(25,26)$.

A key rationale for SBRT is escalation of radiation therapy dose to the tumor. Attempts are made to treat the tumor-vessel abutment region with 50 Gy in 5 fractions. This is equivalent to $84.7 \mathrm{~Gy}$ at the typical fractionation of 1.8 Gy per fraction $(\alpha / \beta=10)$ or the biologically effective dose (BED) of $100 \mathrm{~Gy}_{10}$ suggested for optimal control of early non-small cell lung cancer (27). That dose was not always achievable in our series due to organ at risk limitations. Still, the median dose of 40 Gy in 5 fractions in 
Table 3 Pathologic findings and adjuvant treatments

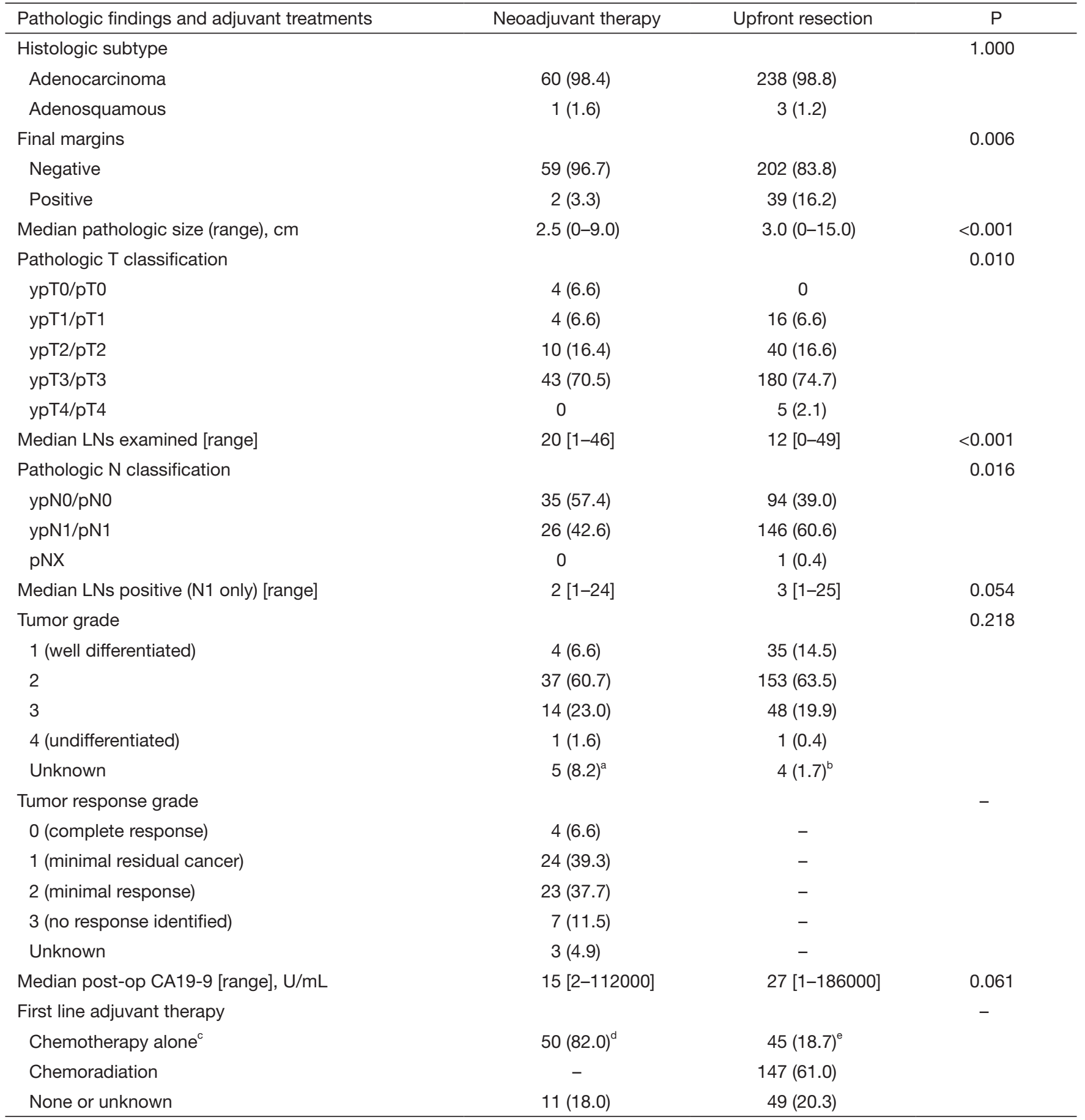

${ }^{\mathrm{a}}$, pathologic complete response or unable to grade from residual isolated tumor cells; ${ }^{\mathrm{b}}$, not reported; ${ }^{\mathrm{c}}$, single-agent gemcitabine in 78 of $95(82.1 \%) ; ~ ', 12$ patients received gemcitabine with an experimental vaccine trial, 1 patient received adjuvant GTX, and 1 other received gemcitabine and cisplatin; ${ }^{e}$, one patient each received erlotinib, gemcitabine and evofosfamide (trial), and FOLFOX. LN, lymph node. 

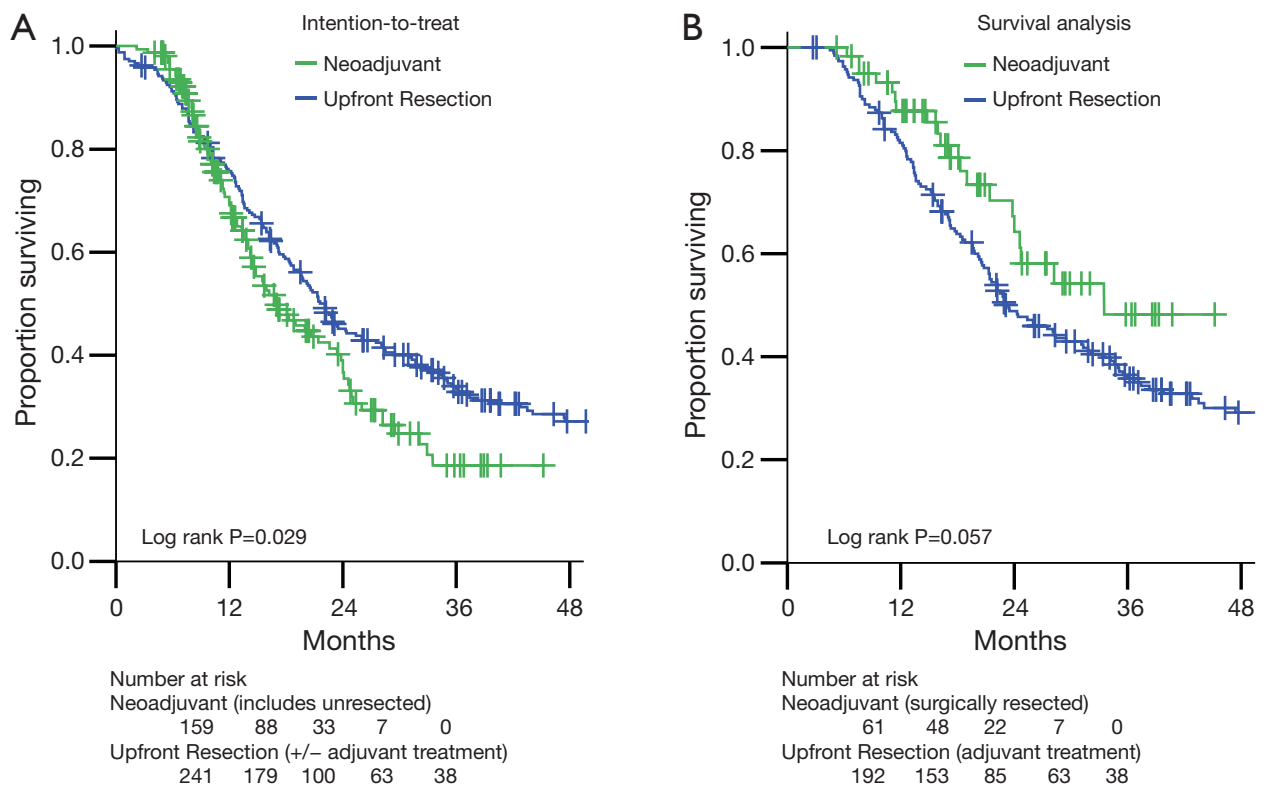

Figure 2 Actuarial overall survival for the cohorts in this study. (A) The intention-to-treat analysis compares the survival of all 241 upfront resectable pancreatic cancer patients who underwent pancreatectomy without neoadjuvant therapy to all 159 patients not judged to be upfront resectable (110 BRPC and 49 LAPC) who underwent neoadjuvant multi-agent chemotherapy and SBRT with the intention of resection. (B) The survival analysis compares the survival of patients who received the intended therapy. There were 192 upfront resection patients who underwent pancreatectomy followed by standard of care chemotherapy or chemoradiotherapy. Sixty-one (56 BRPC and 5 LAPC) patients underwent neoadjuvant multi-agent chemotherapy and SBRT followed by resection. Long-term outcomes after pancreatectomy were not worsened by neoadjuvant therapy. BRPC, borderline resectable pancreatic cancer; LAPC, locally advanced pancreatic cancer; SBRT, stereotactic body radiation therapy.

this study represents a dose escalated equivalent of $61.0 \mathrm{~Gy}$ at 1.8 Gy per fraction. Also, alternative mechanisms of tumor killing have been observed for radiation doses of $\geq 8$ Gy per fraction (28). These benefits are currently theoretical, and this work provides evidence for the safety of applying neoadjuvant multi-agent chemotherapy and SBRT with the goal of curative intent resection in coming randomized trials.

\section{Acknowledgements}

None.

\section{Footnote}

Conflicts of Interest: The authors have no conflicts of interest to declare.

Ethical Statement: The study was approved by the Institutional Review Board of the University of South Florida (No. Pro00003382).

\section{References}

1. Rahib L, Smith BD, Aizenberg R, et al. Projecting cancer incidence and deaths to 2030: the unexpected burden of thyroid, liver, and pancreas cancers in the United States. Cancer Res 2014;74:2913-21.

2. Bilimoria KY, Bentrem DJ, Ko CY, et al. Validation of the 6th edition AJCC Pancreatic Cancer Staging System: report from the National Cancer Database. Cancer 2007;110:738-44.

3. Bilimoria KY, Talamonti MS, Sener SF, et al. Effect of hospital volume on margin status after pancreaticoduodenectomy for cancer. J Am Coll Surg 2008;207:510-9.

4. Callery MP, Chang KJ, Fishman EK, et al. Pretreatment assessment of resectable and borderline resectable pancreatic cancer: expert consensus statement. Ann Surg Oncol 2009;16:1727-33.

5. Springett GM, Hoffe SE. Borderline resectable pancreatic cancer: on the edge of survival. Cancer Control 2008;15:295-307.

6. Assifi MM, Lu X, Eibl G, et al. Neoadjuvant therapy in 
pancreatic adenocarcinoma: a meta-analysis of phase II trials. Surgery 2011;150:466-73.

7. Satoi S, Yanagimoto H, Toyokawa H, et al. Surgical results after preoperative chemoradiation therapy for patients with pancreatic cancer. Pancreas 2009;38:282-8.

8. Estrella JS, Rashid A, Fleming JB, et al. Post-therapy pathologic stage and survival in patients with pancreatic ductal adenocarcinoma treated with neoadjuvant chemoradiation. Cancer 2012;118:268-77.

9. Stokes JB, Nolan NJ, Stelow EB, et al. Preoperative capecitabine and concurrent radiation for borderline resectable pancreatic cancer. Ann Surg Oncol 2011;18:619-27.

10. Massucco P, Capussotti L, Magnino A, et al. Pancreatic resections after chemoradiotherapy for locally advanced ductal adenocarcinoma: analysis of perioperative outcome and survival. Ann Surg Oncol 2006;13:1201-8.

11. White RR, Hurwitz HI, Morse MA, et al. Neoadjuvant chemoradiation for localized adenocarcinoma of the pancreas. Ann Surg Oncol 2001;8:758-65.

12. Araujo RL, Gaujoux S, Huguet F, et al. Does pre-operative chemoradiation for initially unresectable or borderline resectable pancreatic adenocarcinoma increase postoperative morbidity? A case-matched analysis. HPB (Oxford) 2013;15:574-80.

13. Rajagopalan MS, Heron DE, Wegner RE, et al. Pathologic response with neoadjuvant chemotherapy and stereotactic body radiotherapy for borderline resectable and locallyadvanced pancreatic cancer. Radiat Oncol 2013;8:254.

14. Mellon EA, Hoffe SE, Springett GM, et al. Long-term outcomes of induction chemotherapy and neoadjuvant stereotactic body radiotherapy for borderline resectable and locally advanced pancreatic adenocarcinoma. Acta Oncol 2015;54:979-85.

15. Greenblatt DY, Kelly KJ, Rajamanickam V, et al. Preoperative factors predict perioperative morbidity and mortality after pancreaticoduodenectomy. Ann Surg Oncol 2011;18:2126-35.

16. Tsuruga Y, Kamachi H, Wakayama K, et al. Portal vein stenosis after pancreatectomy following neoadjuvant chemoradiation therapy for pancreatic cancer. World J Gastroenterol 2013;19:2569-73.

17. Hoyer M, Roed H, Sengelov L, et al. Phase-II study on stereotactic radiotherapy of locally advanced pancreatic carcinoma. Radiother Oncol 2005;76:48-53.

18. Schellenberg D, Goodman KA, Lee F, et al. Gemcitabine chemotherapy and single-fraction stereotactic body radiotherapy for locally advanced pancreatic cancer. Int J Radiat Oncol Biol Phys 2008;72:678-86.

19. Chuong MD, Springett GM, Freilich JM, et al. Stereotactic body radiation therapy for locally advanced and borderline resectable pancreatic cancer is effective and well tolerated. Int J Radiat Oncol Biol Phys 2013;86:516-22.

20. Chuong MD, Springett GM, Weber J, et al. Induction gemcitabine-based chemotherapy and neoadjuvant stereotactic body radiation therapy achieve high marginnegative resection rates for borderline resectable pancreatic cancer. J Radiat Oncol 2012;1:273-81.

21. Vignesh S, Hoffe SE, Saif MW. EUS-guided pancreatic diagnosis and beyond. Highlights from the "2011 ASCO gastrointestinal cancers symposium". San Francisco, CA, USA. January 20-22, 2011. JOP 2011;12:86-91.

22. Regine WF, Winter KA, Abrams RA, et al. Fluorouracil vs gemcitabine chemotherapy before and after fluorouracilbased chemoradiation following resection of pancreatic adenocarcinoma: a randomized controlled trial. JAMA 2008;299:1019-26.

23. Chun YS, Milestone BN, Watson JC, et al. Defining venous involvement in borderline resectable pancreatic cancer. Ann Surg Oncol 2010;17:2832-8.

24. Takahashi H, Ohigashi H, Gotoh K, et al. Preoperative gemcitabine-based chemoradiation therapy for resectable and borderline resectable pancreatic cancer. Ann Surg 2013;258:1040-50.

25. Park JK, Paik WH, Ryu JK, et al. Clinical significance and revisiting the meaning of CA 19-9 blood level before and after the treatment of pancreatic ductal adenocarcinoma: analysis of 1,446 patients from the pancreatic cancer cohort in a single institution. PLoS One 2013;8:e78977.

26. Boone BA, Steve J, Zenati MS, et al. Serum CA 19-9 response to neoadjuvant therapy is associated with outcome in pancreatic adenocarcinoma. Ann Surg Oncol 2014;21:4351-8.

27. Onishi H, Shirato H, Nagata Y, et al. Hypofractionated stereotactic radiotherapy (HypoFXSRT) for stage I non-small cell lung cancer: updated results of 257 patients in a Japanese multi-institutional study. J Thorac Oncol 2007;2:S94-100.

28. Fuks Z, Kolesnick R. Engaging the vascular component of the tumor response. Cancer Cell 2005;8:89-91.

Cite this article as: Mellon EA, Strom TJ, Hoffe SE, Frakes JM, Springett GM, Hodul PJ, Malafa MP, Chuong MD, Shridhar R. Favorable perioperative outcomes after resection of borderline resectable pancreatic cancer treated with neoadjuvant stereotactic radiation and chemotherapy compared with upfront pancreatectomy for resectable cancer. J Gastrointest Oncol 2016;7(4):547-555. doi: 10.21037/jgo.2016.03.15 


\section{Supplementary}

\section{Supplementary materials}

Univariate and multivariate correlations with overall survival

Univariate analysis (UVA) and multivariate analysis (MVA) for OS was performed by Cox Regression. Due to differences in imaging used for clinical staging between the two groups, clinical stage was not assigned in $40 \%$ of the upfront resection group. As such, comparison T and N classifications were used for UVA and MVA in Table S1, where clinical classification is used in the neoadjuvant therapy group and pathologic classification is used in the upfront resection group.

In the UVA, N1 classification was significant for worse OS $(\mathrm{P}=0.035)$. Sex, tumor location, $\mathrm{T}$ classification, and number of lymph nodes examined were not significant $(\mathrm{P}>0.1)$. Trends towards significance were observed for benefit to younger age $(\mathrm{P}=0.056)$ and the neoadjuvant therapy group $(\mathrm{P}=0.059)$. On MVA, Trends remained for neoadjuvant therapy group $(\mathrm{P}=0.077)$ and $\mathrm{N}$ classification $(\mathrm{P}=0.091)$.

Table S1 Univariate and multivariate analysis of overall survival

\begin{tabular}{|c|c|c|c|c|c|c|}
\hline \multirow{2}{*}{ Parameters } & \multicolumn{3}{|c|}{ Univariate $^{*}$} & \multicolumn{3}{|c|}{ Multivariate } \\
\hline & $\mathrm{HR}$ & $95 \% \mathrm{Cl}$ & $\mathrm{P}$ & $\mathrm{HR}$ & $95 \% \mathrm{Cl}$ & $\mathrm{P}$ \\
\hline Age (years, continuous) & 1.015 & $1.000-1.030$ & 0.056 & 1.012 & $0.997-1.027$ & 0.131 \\
\hline Head location (vs. other) & 0.861 & $0.580-1.278$ & 0.458 & 1.181 & $0.782-1.782$ & 0.429 \\
\hline Comparison & 1.143 & $0.854-1.531$ & 0.368 & 1.160 & $0.855-1.574$ & 0.340 \\
\hline Comparison classification NO (vs. N1) & 0.837 & $0.710-0.987$ & 0.035 & 1.339 & $0.955-1.876$ & 0.091 \\
\hline Lymph nodes examined (continuous) & 0.995 & $0.978-1.013$ & 0.589 & 1.000 & $0.981-1.019$ & 0.970 \\
\hline Neoadjuvant therapy (vs. adjuvant) & 0.796 & $0.627-1.009$ & 0.059 & 0.637 & $0.386-1.051$ & 0.077 \\
\hline No vascular repair (vs. yes) ${ }^{\#}$ & 1.023 & $0.794-1.318$ & 0.861 & - & - & - \\
\hline Margins negative (vs. positive) & 0.888 & $0.719-1.096$ & 0.268 & - & - & - \\
\hline
\end{tabular}

*, patients in the upfront resection group who did not receive adjuvant treatment were excluded from survival analysis; ", included only in the UVA. 